\title{
Temperature Control for a Proton-Exchange Membrane Fuel Cell System with Unknown Dynamic Compensations
}

\author{
Yashan Xing $\mathbb{D},{ }^{1}$ Ramon Costa-Castelló $\mathbb{D},{ }^{1}$ and Jing $\mathrm{Na}^{2}$ \\ ${ }^{1}$ Institut de Robòtica i Informàtica Industrial, CSIC-UPC. C/ Llorens i Artigas 4-6, Barcelona 08028, Spain \\ ${ }^{2}$ Faculty of Mechanical and Electrical Engineering, Kunming University of Science and Technology, Kunming 650500, China \\ Correspondence should be addressed to Ramon Costa-Castelló; ramon.costa@upc.edu
}

Received 6 August 2020; Revised 18 September 2020; Accepted 27 September 2020; Published 23 October 2020

Academic Editor: Zhile Yang

Copyright (c) 2020 Yashan Xing et al. This is an open access article distributed under the Creative Commons Attribution License, which permits unrestricted use, distribution, and reproduction in any medium, provided the original work is properly cited.

Numerous control strategies of temperature regulation have been carried out for proton-exchange membrane fuel cell systems including a cooling fan in order to ensure operation at the desired condition and extend the lifetime of the fuel cell stack. However, most existing control strategies are developed without considering the efficiency limitation of the cooling system such that the cooling fan may be unable to eliminate the additional heat. Moreover, there are unknown modelling errors, external disturbance and noise during modelling and experiment processes for fuel cells. Due to those unknown dynamics, the conventional control strategies may fail to achieve the expectant results. To address this issue, an alternative control strategy is proposed in this paper, which consists of a composite proportional-integral (PI) controller with an unknown system dynamics estimator. First, the control strategy is developed by reducing the temperature of input air through the humidifier and simultaneously increasing the mass flow of air in order to eliminate the excess heat that a cooling fan cannot remove. Moreover, an unknown system dynamics estimator is proposed in order to compensate the effect of the unknown dynamics. The construction of the estimator is designed through finding an invariant manifold which implies the relation between known variables and the unknown manifold. The invariant manifold is derived by applying a simple low-pass filter to the system which is beneficial to avoid the requirement of the unmeasurable state derivative. Furthermore, the proposed estimator is easily merged into the proposed PI control strategy and ensures the exponential convergence of estimated errors. Besides, the estimator is further modified such that the derivative of the desired temperature is not required in the controller. Finally, numerical simulations of the PEMFC system are provided and the results illustrate the efficacy of the proposed control strategy.

\section{Introduction}

Proton-exchange membrane fuel cell (PEMFC) is regarded as one of the cleanest alternative energy conversions which is promising to replace traditional power generation technologies [1]. The energy conversion principle of PEMFC is to transform chemical energy into electricity, thermal energy, and water without requiring any moving part. Due to its properties of low operation temperature and fast start-up compared with other types of fuel cells, it has drawn wide attention in the fields of power station systems and vehicle systems [2, 3]. However, there are numerous stumbling blocks for widely commercial applications $[4,5]$.

PEMFC involves a multiphysics coupling process which leads to some difficulties for control strategy design and implementation. Among those, temperature regulation of PEMFC stack is essential to ensure the high operation efficiency, avoid the thermal fatigue, and prolong the stack lifespan [6, 7]. Several control strategies regarding the temperature regulation have been carried out in recent years. In [8], an extremum seeking control with a proportionalintegral (PI) controller was proposed in order to achieve the optimal temperature regulation. In [9], the temperature control strategy was proposed for a PEMFC system with a water cooling system, where the voltage of a water circulation pump was manipulated through a PI controller, and a water cooling fan was under the on/off modes. In [10], a more advanced fuzzy logic controller was applied to regulate the speed of the cooling fan and increase the convection ratio between PEMFC and atmosphere. However, the proposed 
control strategy is validated for a low-power PEMFC. Thus, the limitation of cooling capacity is not considered in those aforementioned control strategies [8-10]. When the generated heat from PEMFC is higher than the dissipated heat from the cooling system, the temperature of PEMFC will keep increasing. Recently, Chen et al. [11] considered the limited capacity of a water cooling system and current constraint in the PEMFC system and proposed three temperature control strategies, which developed a constrained model predictive control (MPC) strategy, and designed duty ratio split controller and current governor both based on the proportiona-integral-derivative (PID) control in order to implement the trade-off between cooling capacity and power demand.

On the other hand, most control strategies mainly depend on a reliable mathematical model while more accurate models with multiple dimensions can introduce mathematical complexities. Due to those complexities, many control design methodologies cannot be applied. Moreover, the control-oriented model contains unmodelled dynamics and modelling errors due to model simplification process. Besides, external disturbances and noise from the experiment cannot be avoided. Those unknown dynamics may result in unexpected control results and even cause system unstable. Thus, it is significant to develop an estimator or an observer to eliminate the unknown disturbance and enhance the controller performance.

In this line, the disturbance observer (DOB) was first proposed to address the unknown dynamics $[12,13]$. Furthermore, the modified DOB for nonlinear system was designed in [14]. Moreover, the extended state observer (ESO) $[15,16]$ as an alternative method was developed, where the extended states of the unknown dynamics and the initial states were observed simultaneously. In [17], the ESO incorporated into a sliding mode control was used to achieve the temperature regulation in PEMFC. More recently, a modified ESO based on the Kalman-Bucy filter was incorporated into PID control to implement the temperature management for PEMFC in [18]. Besides, a new framework of unknown system dynamics estimator (USDE) [19] was proposed to deal with the unknown dynamics. It has been incorporated in many control strategies and applied in many industrial fields, such as hydraulic servo systems [20], nonlinear robotic systems [21], and spark ignition engines [22]. However, designing a proper control strategy with consideration of unknown dynamics remains as an open and interesting problem for PEMFC.

This paper aims to propose an alternative control strategy of temperature regulation for a PEMFC system with consideration of the cooling fan limitation. Moreover, the unknown dynamic compensation is developed in order to enhance the performance for the proposed controller. To be specific, we first consider the limitation of the fan efficiency, where the maximum energy generated from a cooling fan is not enough to force the stack temperature into the desired value. Due to this limitation, the control strategy is proposed through increasing the mass flow of the input air and reducing the temperature of the humidifier to implement PEMFC temperature management. By considering the practical application, this control strategy is developed based on the PI controller. Furthermore, an unknown system dynamics estimator (USDE) is used in order to compensate the effect of the unknown dynamics. The construction of this estimator is through generating an invariant manifold in order to ensure the exponential convergence of the estimation errors, where the invariant manifold provides the information between known variables and the unknown dynamics. It is noticed that the derived estimator is easily merged into the proposed PI control strategy without introducing any complexity. Since the derivative of the desired temperature is not measurable, the estimator is further modified such that the derivative of the desired temperature is not required in the proposed controller. To summarize, the main contributions of the proposed control strategy are as follows:

(1) The proposed estimator for unknown dynamics is simple and easy to incorporate into the controller through finding an invariant manifold. It is significant for practical application that the construction of the invariant manifold is based on a simple lowpass filter operation without introducing the unmeasurable state derivative. Moreover, only one parameter of the bandwidth for the low-pass filter is required to be tuned.

(2) With consideration of the limited operation property of the cooling fan, the control strategy is proposed through increasing air mass flow and reducing the temperature of input air such that the voltage change is affected less and the desired temperature can be guaranteed simultaneously.

The rest of the paper is organised as follows. In Section 2, the model description of the PEMFC system and the relevant problem formulation are presented. Section 3 and Section 4 illustrate the control strategy for the PEMFC system with respect to the USDE and the composite PI controller. The numerical results of the proposed controller for the PEMFC system are provided in Section 5. Finally, the conclusions are given in Section 6.

\section{Model Description of PEMFC System}

2.1. Model Description. In this section, a PEMFC system is first introduced, and its schematic diagram is depicted in Figure 1. The PEMFC system mainly consists of two humidifiers, two line heaters, a cooling fan, and a fuel cell stack. To begin with, the fuel cell stack is a low-temperature PEMFC with 8 cells with an active area of $50 \mathrm{~cm}^{2}$. The combination of anode, cathode, and electrolyte layer constitutes the core of PEMFC in the sandwich structure, as depicted in Figure 2. With respect to gas transport processes in the PEMFC system, the compressed air is first fed into the cathode channel through a humidifier and a line heater. Due to the material property of electrolyte, the PEMFC stack requires enough humidity for the electrolyte layer. Before entering into the fuel cell stack, the supplied gases need to pass through the humidifier and the line heater in order to guarantee the required humidity and maintain the gas 


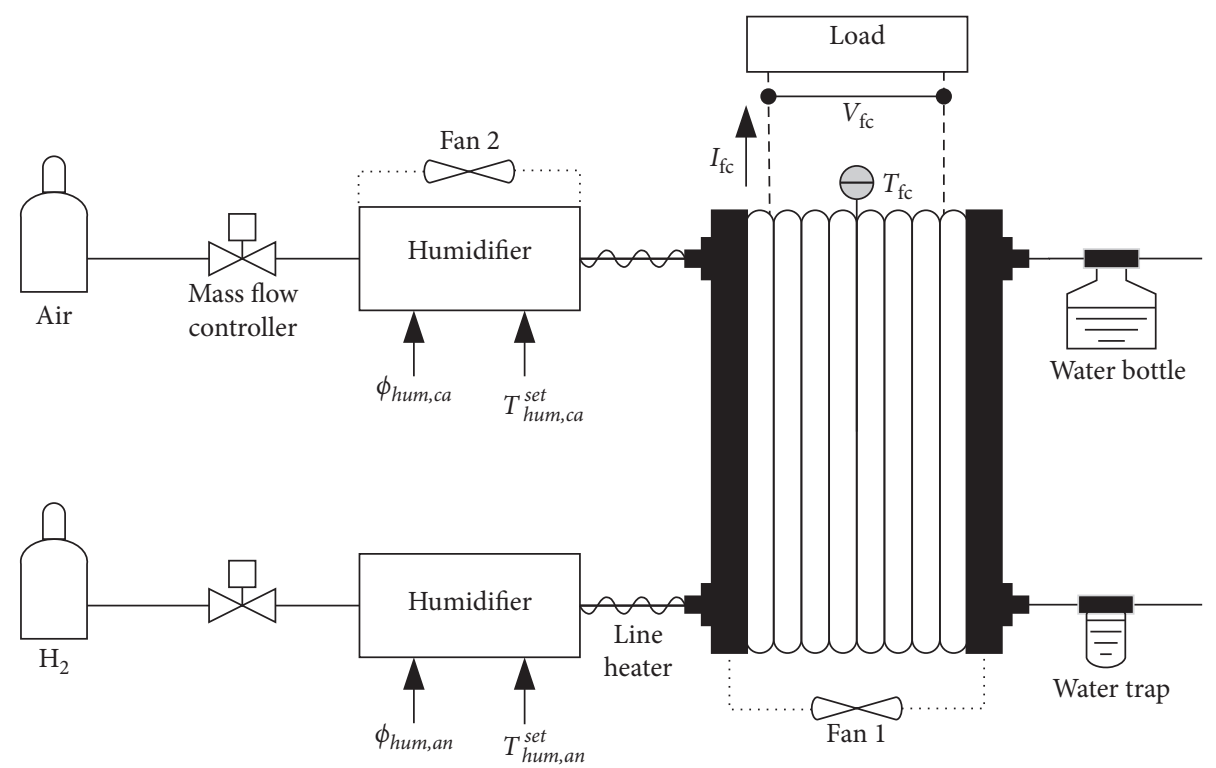

FIgURE 1: Schematic diagram of a PEMFC stack and the PEMFC system.

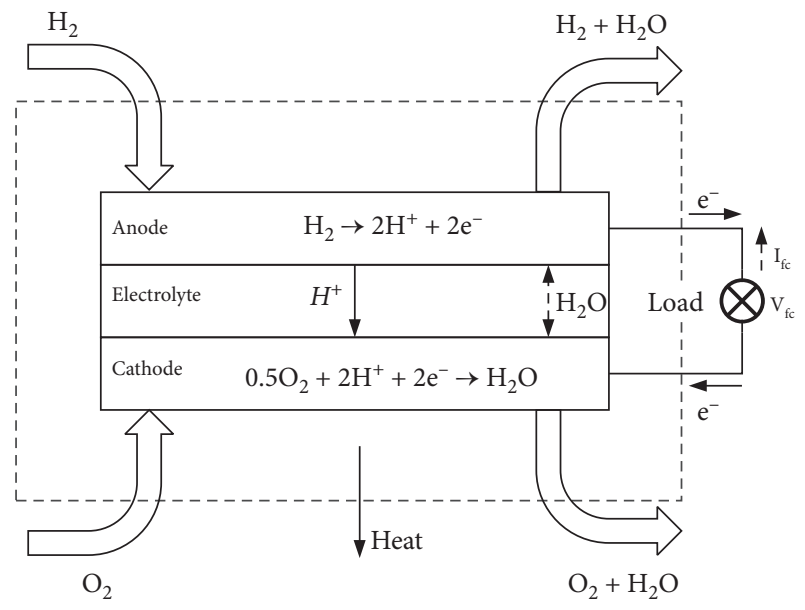

FIgURE 2: The operation principle of the PEMFC stack.

temperature in the pipelines simultaneously. In the anode channel, the pure hydrogen is provided by a hydrogen tank and goes through another humidifier and another line heater such that the hydrogen is injected with some required water vapor. Furthermore, the hydrogen ions can pass through the electrolyte to cathode layer and react with oxygen. During this process, there is some generated water which can affect the humidity in each channel. When the relative humidity of each channel exceeds $100 \%$, the vapor condenses into the liquid form. In this paper, we assume the liquid water cannot leave the stack until it evaporates into a gas form. Finally, the unreacted gases will be exhausted to atmosphere, and the output water vapor will be cooled down and stored in the outside vessel.

For the cooling system, there is a fan (Fan 1 in Figure 1) which works for regulating the PEMFC temperature. In practice, this fan is not efficient enough to regulate the PEMFC temperature when PEMFC operates at the high power condition. Moreover, the humidifier contains internal resistances to heat the injected liquid water and the input gases, while the cooling system is not included in the internal structure of the humidifier. Due to the constraint of this structure, the cooling dynamics of the humidifier is extremely slow. The outside fan (fan 2 in Figure 2) is used to help reduce the humidifier temperature at the cathode side. Therefore, the relevant modelling of the humidifier, the line heater, and PEMFC stack including cooling process of the fan will be presented in the following.

2.1.1. Auxiliary Components. The humidifier is used to ensure the desired relative humidity of input gases. Its main process is that the water in the vapor form is injected into input gases. Thus, the total mass flow of output gases from the humidifier is expressed as

$$
\dot{m}_{\mathrm{hum}, j}^{\text {out }}=\dot{m}_{\mathrm{hum}, j}^{\text {in }}+\dot{m}_{\mathrm{H}_{2} \mathrm{O}, j}^{\text {inj }}
$$

where $j$ represents the anode or cathode channel in PEMFC and $\dot{m}_{\text {hum }, j}^{\text {in }}$ is the input gas flow for the humidifier at the side $j$. In order to achieve the desired relative humidity, the required vapor water injected into the input gases $\dot{m}_{\mathrm{H}_{2} \mathrm{O}, j}^{\text {inj }}$ can be computed as

$$
\dot{m}_{\mathrm{H}_{2} \mathrm{O}, j}^{\text {inj }}=\frac{M_{\mathrm{H}_{2} \mathrm{O}} \phi_{\mathrm{hum}, j} \dot{m}_{\mathrm{hum}, j}^{\text {out }} P_{\mathrm{sat}}\left(T_{\mathrm{hum}, j}\right)}{\bar{M}_{j} P_{\mathrm{hum}, j}^{\text {in }}},
$$

where $\phi_{\text {hum, } j}$ is the desired relative humidity of output gases; $M_{\mathrm{H}_{2} \mathrm{O}}$ is the molar mass of water; The average molar mass of gases for the channel $j$ is denoted as $\vec{M}_{j} ; P_{\text {hum, }}^{\text {in }}$ represents the pressure of input gases. And $P_{\text {sat }}\left(T_{\text {hum }, j}\right)$ is the pressure of vapor saturation at the humidifier temperature $T_{\text {hum, } j}$. Moreover, the saturation pressure $P_{\text {sat }}$ related to the relevant temperature $T_{0}$ is approximately calculated as [23] 


$$
\begin{aligned}
\log _{10}\left(P_{\text {sat }}\right)= & -1.69 \times 10^{-10} T_{0}^{4}+3.85 \times 10^{-7} T_{0}^{3} \\
& -3.39 \times 10^{-4} T_{0}^{2}+0.143 T_{0}-20.92 .
\end{aligned}
$$

The output gases from the humidifier enter into PEMFC stack through a pipeline. The temperature of this pipeline has some effect on the gas pressure and relative humidity. In order to avoid vapor condensation and maintain desired relative humidify of input gases, the line heater is installed on the pipe. Based on Dalton's law, the partial pressure of vapor water at the line heater is calculated as

$$
P_{\mathrm{H}_{2} \mathrm{O}, j}^{\mathrm{lh}}=\frac{T_{\mathrm{lh}, j}}{T_{\mathrm{hum}, j}} P_{\mathrm{H}_{2} \mathrm{O}, j}^{\mathrm{inj}}
$$

where $T_{\mathrm{lh}, j}$ is the temperature of the line heater. The partial pressure of injected vapor is denoted as $P_{\mathrm{H}_{2} \mathrm{O}, j}^{\mathrm{inj}}=\phi_{\text {hum }, j} P_{\text {sat }}\left(T_{\text {hum }, j}\right)$. Moreover, the relative humidity of gases at the line heater is expressed as

$$
\phi_{\mathrm{lh}, j}=\frac{P_{\mathrm{H}_{2} \mathrm{O}, j}^{\mathrm{lh}}}{\phi_{\mathrm{lh}, j} P_{\mathrm{sat}}\left(T_{\mathrm{lh}, j}\right)},
$$

where $P_{\text {sat }}$ is the saturation pressure of vapor at the line heater temperature $T_{\mathrm{lh}, j}$, which can be calculated by (3).

2.1.2. PEMFC Stack. For control design purpose, we assume that the temperature of gases in the stack is the same as the electrolyte structure. The temperature variation along the structure dimension is not considered in this paper. Based on the energy conservation principle, the thermal energy balance of PEMFC is built as follows:

$$
\begin{aligned}
m_{\mathrm{fc}} C_{p, \mathrm{fc}} \frac{\mathrm{d} T_{\mathrm{fc}}}{\mathrm{d} t}= & H_{\mathrm{ca}}^{\mathrm{in}}+H_{\mathrm{an}}^{\mathrm{in}}-H_{\mathrm{ca}}^{\text {out }}-H_{\mathrm{an}}^{\text {out }} \\
& +H_{r}-V_{\mathrm{fc}} I_{\mathrm{fc}}-H_{\mathrm{fan}}+d,
\end{aligned}
$$

where $m_{\mathrm{fc}}$ and $T_{\mathrm{fc}}$ are the mass and the temperature of PEMFC, respectively. The specific heat capacity of PEMFC is denoted as $C_{p, \mathrm{fc}} ; V_{\mathrm{fc}}$ is the output voltage of PEMFC; $d$ is regarded as the unknown disturbance. The input energy values of cathode $H_{\mathrm{ca}}^{\mathrm{in}}$ and anode layers $H_{\mathrm{an}}^{\text {in }}$ generated by the input gases calculated by

$$
\begin{aligned}
H_{\mathrm{ca}}^{\text {in }} & =\sum_{\mathrm{ca}} \frac{w_{i}^{\text {in }}}{M_{i}} \int_{T_{\mathrm{ref}}}^{T_{\mathrm{lh}, \mathrm{ca}}} C_{p, i}(T) \mathrm{d} T, \\
H_{\mathrm{an}}^{\text {in }} & =\sum_{\mathrm{an}} \frac{w_{i}^{\mathrm{in}}}{M_{i}} \int_{T_{\mathrm{ref}}}^{T_{\mathrm{lh}, \mathrm{an}}} C_{p, i}(T) \mathrm{d} T,
\end{aligned}
$$

where $i$ denotes as each species of gases in the cathode or anode layer and $M_{i}$ is the molar mass of the gas $i$. The mass flow rate of gas $i$ is represented as $w_{i}^{\text {in }}$; $T_{\text {ref }}$ is the reference temperature. The specific heat capacity of gas $i$ is denoted as $C_{p, i}$. It can be computed as $C_{p, i}(T)=c_{0, i}+c_{1, i} T+$ $c_{2, i} T^{2}+c_{3, i} T^{3}$, where the heat capacity coefficients $c_{0, i}, c_{1, i}$, $c_{2, i}$, and $c_{3, i}$ can be found in [24].

Furthermore, the output energy of cathode $H_{\text {ca }}^{\text {out }}$, anode $H_{\mathrm{an}}^{\text {out }}$, and the generated energy $H_{r}$ from chemical reaction are expressed as [25]

$$
\begin{aligned}
H_{\mathrm{ca}}^{\mathrm{out}} & =\sum_{\mathrm{ca}} \frac{w_{i}^{\mathrm{out}}}{M_{i}} \int_{T_{\mathrm{ref}}}^{T_{\mathrm{fc}}} C_{p . i}(T) \mathrm{d} T, \\
H_{\mathrm{an}}^{\mathrm{out}} & =\sum_{\mathrm{an}} \frac{w_{i}^{\mathrm{out}}}{M_{i}} \int_{T_{\mathrm{ref}}}^{T_{\mathrm{fc}}} C_{p . i}(T) \mathrm{d} T, \\
H_{r} & =-\frac{w_{H_{2}}^{r}}{M_{H_{2}}} \Delta H_{r}^{o},
\end{aligned}
$$

where $w_{i}^{\text {out }}$ represents the mass flow rate of gas $i$. The mass flow rate of reacted hydrogen is denoted as $w_{H_{2}}^{r} ; M_{\mathrm{H}_{2}}$ is the molar mass of hydrogen. And the specific heat of chemical reaction is represented as $\Delta H_{r}^{o}$.

There is a fan (Fan 1 in Figure 1) close to the PEMFC stack which is used to regulate the stack temperature. The operation principle is that when the stack temperature is higher than the desired temperature, the fan turns on in order to reduce the stack temperature. Otherwise, it turns off. This procedure is expressed as the following mathematical expression:

$$
H_{\text {fan }}= \begin{cases}C_{\text {loss }} A_{\mathrm{fc}}\left(T_{\mathrm{fc}}-T_{\mathrm{ref}}\right), & T_{\mathrm{fc}}>T_{\text {set }}, \\ 0, & T_{\mathrm{fc}} \leq T_{\text {set }},\end{cases}
$$

where $A_{\mathrm{fc}}$ is the surface area of PEMFC; $C_{\text {loss }}$ is the heat transfer constant; and $T_{\text {set }}$ is the desired temperature of the stack.

In the PEMFC, the potential difference is generated by ion flow transports from the electrochemical reaction. The theoretical voltage of PEMFC can change reversibly, which is affected by the variation of partial pressures for reactants and products. Thus, the theoretical voltage at the nonstandard condition can be determined by the Nernst equation, that is,

$$
V_{N}=V_{N 0}+\frac{\Delta s}{2 F}\left(T_{\mathrm{fc}}-T_{\text {ref }}\right)+\frac{R T_{\mathrm{fc}}}{2 F}\left[\ln \left(P_{\mathrm{H}_{2}}\right)+\frac{1}{2} \ln \left(P_{\mathrm{O}_{2}}\right)\right] \text {, }
$$

where $V_{N 0}$ is the theoretical voltage at the standard condition; $P_{\mathrm{H}_{2}}$ and $P_{\mathrm{O}_{2}}$ are partial pressures of hydrogen and oxygen, respectively; $\Delta s$ is the correction coefficient related to the entropy of chemical reaction; $F$ is Faraday's constant; and $R$ denotes the gas constant.

Moreover, there are three types of voltage losses which can affect the voltage variation irreversibly during operation processes. Considering those voltage losses, the output voltage of PEMFC is expressed by [23]

$$
V_{\text {fc }}=n \cdot\left(V_{N}-V_{\text {act }}-V_{\text {ohm }}-V_{\text {con }}\right),
$$

where $n$ is the number of cells for PEMFC; $V_{\text {act }}$ is the activation losses; $V_{\text {ohm }}$ denotes the ohmic losses; and $V_{\text {con }}$ represents the concentration losses. They are calculated as follows:

$$
\begin{aligned}
& V_{\mathrm{act}}=V_{\mathrm{act}, 0}+K_{\mathrm{act}}\left(1-e^{-c_{1} i}\right), \\
& V_{\mathrm{ohm}}=\frac{\delta_{m} I_{\mathrm{fc}}}{\eta_{m} A_{\mathrm{fc}}}
\end{aligned}
$$




$$
V_{\text {con }}=I_{\mathrm{fc}}\left(c_{2} \frac{I_{\mathrm{fc}}}{I_{\max }}\right)^{c_{3}}
$$

where $V_{\text {act, } 0}$ is the initial activation voltage drop; The membrane thickness is denoted as $\Delta m ; K_{\mathrm{act}}, c_{1}, c_{2}$, and $c_{3}$ are the empirical constants, which depend on gas partial pressure and temperature; $I_{\max }$ is the maximum current; and $\eta_{m}$ denotes the membrane conductivity, which is related to the membrane water content $\lambda_{m}$ and stack temperature. It can be calculated as

$$
\eta_{m}=b_{1} e^{b_{2}\left(1 / T_{0}-1 / T_{\mathrm{fc}}\right)},
$$

where $T_{0}=303 \mathrm{~K}$ is the reference temperature for membrane conductivity test. The empirical value related to the membrane water content is represented as $b_{1}=b_{11} \lambda_{m}-b_{12}$ and constants $b_{11}, b_{12}$, and $b_{2}$ are determined empirically. Those empirical constants have been discussed in [23].

For the mass flow transports, the detailed model can be found in [23]. The reacted mass flows of hydrogen and oxygen and the generated mass flow of water are calculated based on the electrochemical relationship:

$$
\begin{aligned}
w_{\mathrm{H}_{2}}^{r} & =\frac{N M_{\mathrm{H}_{2}} I_{\mathrm{fc}}}{2 F}, \\
w_{\mathrm{O}_{2}}^{r} & =\frac{N M_{\mathrm{O}_{2}} I_{\mathrm{fc}}}{4 F}, \\
w_{\mathrm{H}_{2} \mathrm{O}}^{g} & =\frac{N M_{\mathrm{H}_{2} \mathrm{O}} I_{\mathrm{fc}}}{2 F},
\end{aligned}
$$

where $M_{\mathrm{O}_{2}}$ and $M_{\mathrm{H}_{2} \mathrm{O}}$ are the molar mass of oxygen and water. It is noticed that the reacted mass flows are directly related to the current $I_{\mathrm{fc}}$. Moreover, the input mass flows are computed as

$$
\begin{aligned}
& w_{\mathrm{H}_{2}}^{\text {in }}=K_{\mathrm{H}_{2}} w_{\mathrm{H}_{2}}^{r}, \\
& w_{\mathrm{O}_{2}}^{\text {in }}=K_{\mathrm{O}_{2}} w_{\mathrm{O}_{2}}^{r},
\end{aligned}
$$

where $K_{\mathrm{H}_{2}}$ and $K_{\mathrm{O}_{2}}$ are the stoichiometric ratios. Thus, the input mass flows can be determined by the desired stoichiometric ratio and the current.

2.2. Problem Formulation. For the PEMFC system shown in Figure 1, it has been well recognized that the fan 1 can be used to regulate the temperature of the fuel cell stack. However, the fan 1 has not enough power to regulate the fuel cell temperature when it operates at relative high power. Without introducing extra cooling systems, reducing the temperature and increasing mass flows of input gases are possible ways to achieve the PEMFC temperature regulation. Besides, some parameters in the mathematical model are empirical values which can lead to some modelling errors. Furthermore, there are unmodelled dynamics derived from the model simplification during the modelling procedure and external disturbances resulted from the experiment measurement. However, ignoring those unknown dynamics and modelling errors may cause the system performance degradation or even instability when the controllers apply to the PEMFC system. Thus, this paper will develop a novel control strategy in order to address both temperature regulation problem and the unknown dynamic compensations.

In the mathematical model of the PEMFC system, there are many empirically determined variables. The value of those variables may change during the fuel cell operation such that the real performance cannot be precisely presented in the mathematical model. One of the most important variables for the thermal energy balance model is the empirical specific heat of chemical reaction $\Delta H_{r}^{o}$, which affects the generated energy $H_{r}$ in the PEMFC. Moreover, there are some noises or disturbances $d$ from the experiment. Due to those facts, we consider the reacted heat $H_{r}$ and disturbances $d$ as the unknown dynamics $D_{1}$, which can be expressed as follows:

$$
D_{1}=H_{r}+d .
$$

From the thermal energy balance of PEMFC in (6), reducing the input energy and increasing the output energy can further achieve the temperature regulation when the energy of the cooling fan reaches the maximum. Those energies are mainly related to the input mass flow rates and the temperature of input flows, which can be adjusted by the humidifier and the mass flow controller. Without the internal cooling system, the dynamics of reducing the humidifier temperature is extremely slow. The fan 2 is used to improve the cooling process of the humidifier. However, the cooling performance of the humidifier with the fan 2 is still slow. Due to the slow dynamics, increasing the input mass flow is considered to extract the excess heat from the PEMFC stack. Furthermore, considering the economic costs, the input mass flow and the input temperature of air are chosen to regulate the temperature of PEMFC in this paper. For the control design purpose, the total extract heat by employing the additional mass flow of air is calculated as follows:

$$
Q_{\text {loss }}=-\Delta w_{\text {air }}^{\text {in }}\left(T_{\text {hum,ca }}^{\text {set }}-T_{\mathrm{fc}}\right) \text {, }
$$

where $\Delta w_{\text {air }}^{\text {in }}$ is the additional mass flow of air and $T_{\text {hum,ca }}^{\text {set }}$ is an ideal operation point for the temperature of cathode humidifier. Therefore, the extracted heat $Q_{\text {loss }}$ is considered as the control input. By the simple algebraic calculus, the total mass flow of the air is the sum of the initial mass flow and the additional mass flow, which is the real control input in practice. Thus, the thermal energy balance of PEMFC with the control input $u=Q_{\text {loss }}$ is expressed as

$$
\begin{aligned}
m_{\mathrm{fc}} C_{p, \mathrm{fc}} \frac{\mathrm{d} T_{\mathrm{fc}}}{\mathrm{d} t}= & H_{\mathrm{ca}, 0}^{\mathrm{in}}+H_{\mathrm{an}}^{\mathrm{in}}-H_{\mathrm{ca}, 0}^{\text {out }}-H_{\mathrm{an}}^{\text {out }} \\
& +H_{r}-V_{\mathrm{fc}} I_{\mathrm{fc}}-H_{\mathrm{fan}}+d+u,
\end{aligned}
$$

where $H_{\mathrm{ca}, 0}^{\mathrm{in}}$ and $H_{\mathrm{ca}, 0}^{\text {out }}$ are the initial heat of input and output energy for the cathode layer. Furthermore, (20) is rewritten as follows for analysis purpose:

$$
\frac{\mathrm{d} T_{\mathrm{fc}}}{\mathrm{d} t}=\frac{1}{m_{\mathrm{fc}} C_{p, \mathrm{fc}}}\left(u+D_{1}+F\right),
$$


where $F=H_{\mathrm{ca}, 0}^{\mathrm{in}}+H_{\mathrm{an}}^{\mathrm{in}}-H_{\mathrm{ca}, 0}^{\text {out }}-H_{\mathrm{an}}^{\text {out }}-V_{\mathrm{fc}} I_{\mathrm{fc}}-H_{\mathrm{fan}}$ is the known nonlinear function.

To summarize, this paper aims to propose an alternative control strategy for a high-power PEMFC, where the cooling system cannot regulate the PEMFC temperature. The control strategy is available when the cooling fan turns on, which consists of a composite PI controller with unknown system dynamic estimator (USDE) in order to achieve the temperature regulation and eliminate the effect of the unknown dynamics $D_{1}$ through the measurable output $T_{\mathrm{fc}}$ and the known functions $F$. The schematic block of the proposed control strategy is shown in Figure 3.

Before we illustrate the control strategy with the USDE, the following assumptions are given with respect to the thermal energy balance of PEMFC (21).

Assumption 1. The temperature $T_{\mathrm{fc}}$ and the control input $u$ are measurable and bounded. And the function $F$ is computable and bounded.

Assumption 2. The unknown dynamics of $D_{1}$ is continuous and its derivative is bounded such that $\left\|\dot{D}_{1}\right\| \leq \eta$.

Remark 1. It is easy to measure the variables of stack temperature $T_{\mathrm{fc}}$, the current $I_{\mathrm{fc}}$, the voltage $V_{f c}$, and the input mass flows for each channel. Thus, the PEMFC can fulfill the aforementioned Assumption 1 trivially. Besides, the derivative of unknown dynamics $\dot{D}_{1}$ in Assumption 2 is not required to be known, which is used for the stability analysis of the proposed controller.

\section{Controller Design of PEMFC System}

In this section, we first present the USDE for $D_{1}$. Then, it will be incorporated into the PI controller. The stability analysis for USDE and the composite PI controller will be provided separately.

3.1. Estimator of Unknown Dynamics. In order to derive an invariant manifold, a low-pass filter operation is applied to the variables $T_{\mathrm{fc}}, F$ and $u$. The filtered variables $T_{\mathrm{fc}, f}, F_{f}$ and $u_{f}$ are expressed as follows:

$$
\begin{cases}\kappa \dot{T}_{\mathrm{fc}, f}+T_{\mathrm{fc}, f}=T_{\mathrm{fc}}, & T_{\mathrm{fc}, f}(0)=0, \\ \kappa \dot{F}_{f}+F_{f}=F, & F_{f}(0)=0, \\ \kappa \dot{u}_{f}+u_{f}=u, & u_{f}(0)=0,\end{cases}
$$

where $\kappa>0$ is a filter constant, which is the only parameter that needs to be tuned for USDE.

Then, the invariant manifold is provided in the following lemma, which illustrates the relation between the unknown dynamics $D_{1}$ with the measurable parameter $T_{\mathrm{fc}}$ and the filtered variables $T_{\mathrm{fc}, f}, F_{f}$, and $u_{f}$.

Lemma 1. From the thermal energy balance of PEMFC in (21) and filtered vectors in (22), we can derive the following manifold: $\lim _{t \longrightarrow \infty}\left[\lim _{\kappa \longrightarrow 0}\left(\frac{T_{\mathrm{fc}}-T_{\mathrm{fc}, f}}{\kappa}-\frac{1}{m_{\mathrm{fc}} C_{p, \mathrm{fc}}}\left(u_{f}+F_{f}+D_{1}\right)\right)\right]=0$.

For any small constant $\kappa>0$, it is invariant and exponentially attractive for the derived manifold (23).

Proof. The auxiliary coordinate for analyzing the manifold invariant is defined as

$$
z_{1}(t)=\frac{T_{\mathrm{fc}}-T_{\mathrm{fc}, f}}{\kappa}-\frac{1}{m_{\mathrm{fc}} C_{p, \mathrm{fc}}}\left(u_{f}+F_{f}+D_{1}\right) .
$$

By differentiating (24), we can derive that

$$
\begin{aligned}
\dot{z}_{1} & =\frac{\dot{T}_{\mathrm{fc}}-\dot{T}_{\mathrm{fc}, f}}{\kappa}-\frac{1}{m_{\mathrm{fc}} C_{p, \mathrm{fc}}}\left(\dot{u}_{f}+\dot{F}_{f}+\dot{D}_{1}\right) \\
& =-\frac{1}{\kappa}\left(z_{1}+\frac{\kappa}{m_{\mathrm{fc}} C_{p, \mathrm{fc}}} \dot{D}_{1}\right) .
\end{aligned}
$$

Then, we select $V_{z_{1}}=\left(z_{1}^{2} / 2\right)$ as Lyapunov function to show the bound of $z_{1}$. Thus, the time derivative of $V_{z_{1}}$ is

$$
\dot{V}_{z_{1}}=z_{1} \dot{z}_{1}=-\frac{1}{\kappa} z_{1}^{2}-\frac{1}{m_{\mathrm{fc}} C_{p, \mathrm{fc}}} z_{1} \dot{D}_{1}
$$

From Assumption 2 and Young's inequality, (26) can be further be derived as

$$
\dot{V}_{z_{1}} \leq-\frac{1}{2 \kappa} z_{1}^{2}+\frac{\kappa}{2 m_{\mathrm{fc}}^{2} C_{p, \mathrm{fc}}^{2}} \dot{D}_{1}^{2} \leq-\frac{1}{\kappa} V_{z_{1}}+\frac{\kappa}{2 m_{\mathrm{fc}}^{2} C_{p, \mathrm{fc}}^{2}} \eta^{2} .
$$

Furthermore, we apply the integration into (27) and we can obtain $V_{z_{1}}(t) \leq\left(e^{-(t / \kappa)} V_{z_{1}}(0)+\kappa^{2} \eta^{2} /\left(2 m_{\mathrm{fc}}^{2} C_{p, \mathrm{fc}}^{2}\right)\right)$. Thus, it is concluded that $z(t)$ converges exponentially to a small compact around zero, which is expressed as

$$
\left\|z_{1}(t)\right\| \leq \sqrt{z_{1}^{2}(0) e^{(-t / \kappa)}+\frac{\kappa^{2} \eta^{2}}{2 m_{\mathrm{fc}}^{2} C_{p, \mathrm{fc}}^{2}}} .
$$

Based on Assumption 2, the upper bound of $\dot{D}_{1}$ is $\eta$. Due to this fact, (28) implies that $\lim _{t \rightarrow \infty} z_{1}(t)=\left(\kappa \eta / m_{\mathrm{fc}} C_{p, \mathrm{fc}}\right)$. Then, we can further obtain that $\kappa \dot{D}_{1} \longrightarrow 0$ and $\kappa^{2} \eta^{2} \longrightarrow 0$ as $\kappa \longrightarrow 0$. Thus, $z_{1}(t)$ will converge to zero for $\kappa \longrightarrow 0$ and/ or $\eta \longrightarrow 0$. Finally, the manifold $z_{1}=0$ in (23) is invariant and exponentially attractive.

Based on the rendered manifold in (23), the estimator for unknown dynamics $D_{1}$ can be designed as

$$
\widehat{D}_{1}=\frac{m_{\mathrm{fc}} C_{p, \mathrm{fc}}}{\kappa}\left(T_{\mathrm{fc}}-T_{\mathrm{fc}, f}\right)-u_{f}-F_{f}
$$

The following theorem is summarized the convergence of the estimator errors for the proposed estimator in (29).

Theorem 1. The estimator (29) is used to obtain the unknown dynamics $D_{1}$ in the thermal energy balance of PEMFC 


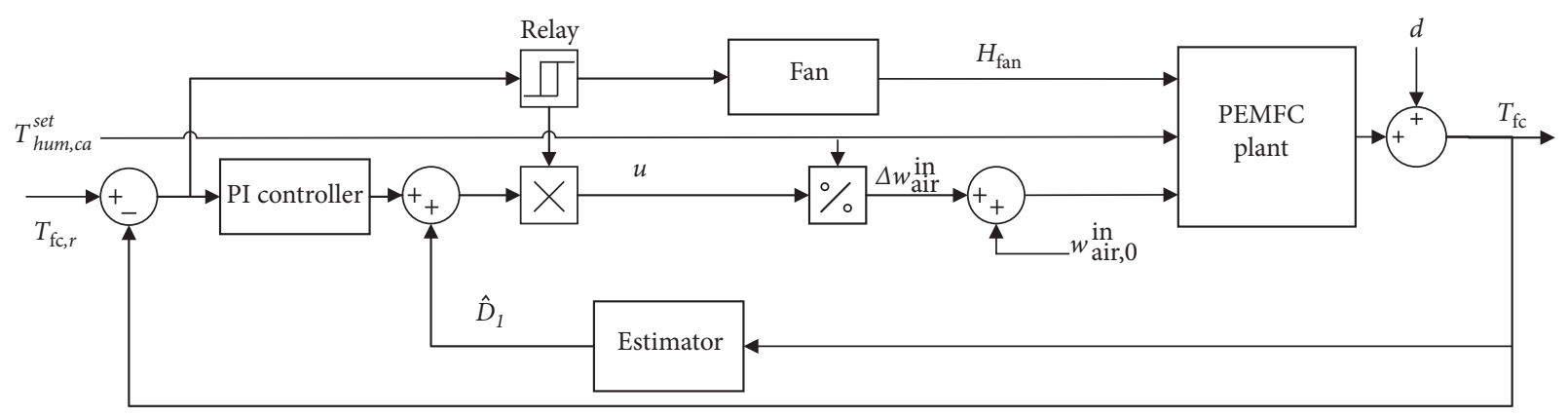

FIgURE 3: Schematic block of the composite PID controller.

(21). The estimation error $\widetilde{D}_{1}=D_{1}-\widehat{D}_{1}$ will exponentially converge to a small compact set around zero.

Proof. To avoid requiring the information of temperature derivative $\dot{T}_{f c}$, we first apply a low-pass filter to the equation (21). Then, we get

$$
\frac{\kappa}{\kappa s+1}\left\{T_{\mathrm{fc}}\right\}=\frac{1}{m_{\mathrm{fc}} C_{p, \mathrm{fc}}}\left(\frac{1}{\kappa s+1}\{u\}+\frac{1}{\kappa s+1}\{F\}+\frac{1}{\kappa s+1}\left\{D_{1}\right\}\right)
$$$$
\& 9 ; \quad+\varepsilon_{1},
$$

where $\varepsilon_{1}$ is the exponentially vanishing term from the filtered initial value of state $T_{\mathrm{fc}}(0)$, which can be neglected.

Moreover, the filtered variable $D_{1, f}$ is defined as

$$
\begin{gathered}
\kappa \dot{D}_{1, f}+D_{1, f}=D_{1}, \\
D_{1, f}(0)=0 .
\end{gathered}
$$

Since the effect of the exponentially vanishing term $\varepsilon$ is not considered, the equation (30) can be rewritten as the following form based on (22) and (31):

$$
\dot{T}_{\mathrm{fc}, f}=\frac{T_{\mathrm{fc}}-T_{\mathrm{fc}, f}}{\kappa}=\frac{1}{m_{\mathrm{fc}} C_{p, \mathrm{fc}}}\left(u_{f}+F_{f}+D_{1, f}\right) .
$$

From the comparison between (24) and (32), it is implied that $\widehat{D}_{1}=D_{1, f}$. Hence, we compute the derivative of the estimation error $\widetilde{D}_{1}$, that is,

$$
\dot{\widetilde{D}}_{1}=\dot{D}_{1}-\dot{\widehat{D}}_{1}=\dot{D}_{1}-\dot{D}_{1, f}=\dot{D}_{1}-\frac{D-D_{1, f}}{\kappa}=-\frac{1}{\kappa} \widetilde{D}_{1}+\dot{D}_{1} .
$$

To show the boundedness of the estimation error $\widetilde{D}_{1}$, we choose Lyapunov function as $V_{D_{1}}=\left(\widetilde{D}_{1}^{2} / 2\right)$. Its derivative is computed as

$$
\dot{V}_{D_{1}}=\widetilde{D}_{1} \dot{\widetilde{D}}_{1}=-\frac{1}{\kappa} \widetilde{D}_{1}^{2}+\widetilde{D}_{1} \dot{D}_{1} .
$$

Considering Young's inequality and Assumption 2, we get

$$
\dot{V}_{D_{1}} \leq-\frac{1}{2 \kappa} \widetilde{D}_{1}+\frac{\kappa}{2} \dot{D}_{1}^{2} \leq-\frac{1}{\kappa} V_{D_{1}}+\frac{\kappa}{2} \eta^{2} .
$$

Then, the integration of (35) is $V_{D} \leq$ $\left(e^{(-t / \kappa)} V_{D_{1}}(0)+\kappa^{2} \eta^{2} / 2\right)$. Based on this, it can be derived that the estimation error $\widetilde{D}_{1}$ will exponentially converge to a small compact:

$$
\left\|\widetilde{D}_{1}\right\| \leq \sqrt{\widetilde{D}_{1}^{2}(0) e^{-t / \kappa}+\kappa^{2} \eta^{2}} .
$$

Finally, $\widetilde{D}_{1}$ will converge to zero when $\kappa \longrightarrow 0$ and $\eta \longrightarrow \infty$

Remark 2. For the unknown system dynamics estimator (29), there are two main advantages in comparison with other estimation (i.e., $[14,26])$. First, only one parameter $\kappa$ is required to be tuned. From (22) and (31), $\kappa$ determines the bandwidth of the low-pass filter such that it has an impact on robustness. Moreover, the boundedness of the unknown dynamics derivative $\eta$ can affect the estimation performance while the constant $\kappa$ can be used to reduce the effect of this boundedness $\eta$. Besides, the convergence speed of the estimation error is related to this constant $\kappa$, which can be found in the proof of Theorem 1. Hence, the selection of this parameter $\kappa$ need to consider the trade-off between the convergence speed of the estimation error and the robustness against disturbance. Moreover, the information of the state derivative $\dot{T}_{\mathrm{fc}, f}$ is not required due to the low-pass filter operations. The discontinuities and chattering phenomena can be avoided compared with the sliding mode-based estimator.

3.2. Composite PI Controller with Unknown Dynamic Compensation. In this section, a composite PI controller based on the USDE is presented. From Theorem 1, the estimator (29) will exponentially converge to a small compact, even to zero when $\kappa \longrightarrow 0$. Hence, it can be used to compensate the unknown dynamics and enhance the control performance. Based on the framework of controller in Figure 3, a composite PI control is designed as

$$
u=Q_{\text {loss }}=k_{p} e_{r}+k_{i} \int_{0}^{t} e_{r}(\tau) \mathrm{d} \tau-\left(\widehat{D}_{1}+F-m_{f c} C_{p, \mathrm{fc}} \dot{T}_{\mathrm{fc}, r}\right),
$$

where $T_{\mathrm{fc}, r}$ denotes the desired temperature of PEMFC; $e_{r}=$ $T_{\mathrm{fc}, r}-T_{\mathrm{fc}}$ is the control error; and the proportional and integral gains are represented as $k_{p}>0$ and $k_{i}>0$, respectively. Since the dynamics of PEMFC temperature is slow, the desired temperature is usually considered as a constant 
or slow-varying value. Thus, the derivative of the desired temperature $\dot{T}_{\mathrm{fc}, r}$ can be neglected.

Remark 3. The proposed control (37) is combined with the estimator of unknown dynamics (29), which has the function to compensate the unknown dynamics. The PI controller is widely used for the PEMFC system in practice. Hence, the proposed control strategy has an advantage to enhance the predesigned PI controller for practical applications.

Moreover, the convergence of the tracking error and the estimation error for the proposed controller are summarized in the following theorem.

Theorem 2. To regulate the temperature of the PEMFC system, the control strategy of the composite PI controller (37) combined with an unknown dynamic compensation is proposed as depicted in Figure 3. The control error $e_{r}$ and the estimation $\widetilde{D}_{1}$ will exponentially converge to a small compact set around zero.

Proof. Based on the thermal balance equation (21) and the controller (37), the derivative of control error is calculated as

$$
\begin{aligned}
\dot{e}_{r} & =\dot{T}_{\mathrm{fc}, r}-\dot{T}_{\mathrm{fc}}=\dot{T}_{\mathrm{fc}, r}-\frac{1}{m_{\mathrm{fc}} C_{p, \mathrm{fc}}} u-\frac{1}{m_{\mathrm{fc}} C_{p, \mathrm{fc}}} D_{1}-\frac{1}{m_{\mathrm{fc}} C_{p, \mathrm{fc}}} F \\
& =-\frac{1}{m_{\mathrm{fc}} C_{p, \mathrm{fc}}}\left(k_{p} e_{r}+k_{i} e_{r i}+\widetilde{D}_{1}\right),
\end{aligned}
$$

where $e_{\mathrm{ri}}=\int_{0}^{t} e_{r}(\tau) \mathrm{d} \tau$ denotes the integral error. To analyze the stability of the controller, the error including the control error and the integral error is defined as $e=\left[e_{\mathrm{ri}} e_{r}\right]^{T}$. Thus, the equation (38) is reformulated as

$$
\begin{aligned}
& \dot{e}=\left[\begin{array}{c}
\dot{e}_{\mathrm{ri}} \\
\dot{e}_{r}
\end{array}\right]=\left[\begin{array}{cc}
0 & 1 \\
-\frac{k_{i}}{m_{\mathrm{fc}} C_{p, \mathrm{fc}}} & -\frac{k_{p}}{m_{\mathrm{fc}} C_{p, \mathrm{fc}}}
\end{array}\right]\left[\begin{array}{l}
e_{\mathrm{ri}} \\
e_{r}
\end{array}\right]+\left[\begin{array}{c}
0 \\
-\frac{1}{m_{\mathrm{fc}} C_{p, \mathrm{fc}}}
\end{array}\right] \widetilde{D}_{1} \\
& =A_{1} e+B_{1} \widetilde{D}_{1} \text {. }
\end{aligned}
$$

In the matrix $A_{1}$, parameters $m_{\mathrm{fc}}$ and $C_{p, \mathrm{fc}}$ and control gains $k_{i}$ and $k_{p}$ are positive constants. In order to guarantee the stability of the control system, the control gains $k_{i}$ and $k_{p}$ are chosen such that the matrix $A_{1}$ is a Hurwitz matrix. According to the Lyapunov theory, there are symmetric and positive definite matrices $P_{1}>0$ and $Q_{1}>0$ such that the matrix $A_{1}$ satisfies the equation $P_{1} A_{1}+A_{1}^{T} P_{1}=-Q_{1}$. Moreover, we choose the Lyapunov function as $V_{e}=$ $\left(\left(e^{T} P_{1} e / 2\right)+\left(\widetilde{D}_{1}^{2} / 2\right)\right)$. Then, its derivative is expressed as

$$
\begin{aligned}
\dot{V}_{e} & =\frac{1}{2} e^{T} P_{1} \dot{e}+\frac{1}{2} \dot{e}^{T} P_{1} e+\widetilde{D}_{1} \dot{\widetilde{D}}_{1} \\
& =\frac{1}{2} e^{T} P_{1} A_{1} e+\frac{1}{2} e^{T} A_{1}^{T} P_{1} e+e^{T} P_{1} B \widetilde{D}_{1}+\widetilde{D}_{1} \dot{\tilde{D}}_{1} \\
& =-\frac{1}{2} e^{T} Q_{1} e+e^{T} P_{1} B \widetilde{D}_{1}-\frac{1}{\kappa} \widetilde{D}_{1}^{2}+\widetilde{D}_{1} \dot{D}_{1}
\end{aligned}
$$

Based on Young's inequality with a positive constant $m>0$, we can further derive that

$$
\begin{aligned}
\dot{V}_{e} & \leq-\frac{1}{2}\left(\lambda_{\min }\left\{Q_{1}\right\}-\frac{m}{2}\right)\|e\|^{2}-\left(\frac{1}{\kappa}-\frac{1+\left\|P_{1} B\right\|^{2}}{2 m}\right) \widetilde{D}_{1}^{2}+\frac{m \eta^{2}}{2} \\
& \leq-\psi_{1} V+\zeta_{1},
\end{aligned}
$$

where there are positive constants of $\psi_{1}=$ $\min \left\{\lambda_{\min }\left\{Q_{1}\right\}-(m / 2) / \lambda_{\max }\left\{P_{1}\right\}, \quad 2\left(1 / \kappa-\left(1+\left\|P_{1} B\right\|^{2}\right)\right) /\right.$ $(2 m)\}$ and $\zeta_{1}=\left(m \eta^{2} / 2\right)$ when constants $m$ and $\kappa$ are satisfied, $m \leq 2 \lambda_{\min }\left\{Q_{1}\right\}$ and $\kappa \leq 2 m /\left(1+\left\|P_{1} B\right\|^{2}\right)$; the maximum eigenvalue of the matrix $P_{1}$ is denoted as $\lambda_{\max }\left\{P_{1}\right\}$; And $\lambda_{\min }\left\{Q_{1}\right\}$ is the minimum eigenvalue of the matrix $Q_{1}$. Then, we can further derive that $V(t) \leq e^{-\psi_{1} t} V(0)+\zeta_{1} / \psi_{1}$, which implies that the control error $e$ and the estimation error $\widetilde{D}_{1}$ will exponentially converge to the following small compact set around zero.

$$
\Omega:=\left\{e, \widetilde{D}_{1} \mid\|e\| \leq \sqrt{\frac{\zeta_{1}}{\psi_{1}}},\left\|\widetilde{D}_{1}\right\| \leq \sqrt{\frac{\zeta_{1}}{\psi_{1}}}\right\} .
$$

\section{Alternative Controller Design of PEMFC System}

In Section 3.2, the controller requires the information of the desired temperature derivative $\dot{T}_{\mathrm{fc}, r}$. Since the desired temperature of the PEMFC system is usually a constant or slow time-varying value, the influence of the desired temperature derivative $\dot{T}_{\mathrm{fc}, r}$ has a small impact on the proposed controller. In order to further eliminate this small impact, an alternative composite PI controller is proposed to further avoid requiring the derivation of the desired temperature $\dot{T}_{\mathrm{fc}, r}$ in this section.

4.1. Alternative Estimator of Unknown Dynamics. Before we present the USDE, we first calculate the derivative of control error in order to find the relation containing the derivative of the desired temperature. Thus, the derivative of control error is computed as

$$
\begin{aligned}
\dot{e}_{r} & =\dot{T}_{\mathrm{fc}, r}-\dot{T}_{\mathrm{fc}}=\dot{T}_{\mathrm{fc}, r}-\frac{1}{m_{\mathrm{fc}} C_{p, \mathrm{fc}}} u-\frac{1}{m_{\mathrm{fc}} C_{p, \mathrm{fc}}} D_{1}-\frac{1}{m_{\mathrm{fc}} C_{p, \mathrm{fc}}} F \\
& =D_{2}\left(\dot{T}_{\mathrm{fc}, r}, D_{1}\right)-\frac{1}{m_{\mathrm{fc}} C_{p, \mathrm{fc}}} u-\frac{1}{m_{\mathrm{fc}} C_{p, \mathrm{fc}}} F
\end{aligned}
$$

where $D_{2}$, including $\dot{T}_{\mathrm{fc}, r}$ and $D_{1}$, is considered as a new unknown dynamics in this section. For analysis, we assume this unknown dynamics is continuous and its derivative is bounded such that $\left\|\dot{D}_{2}\right\| \leq \eta_{2}$. Therefore, $\dot{T}_{\mathrm{fc}, r}$ and $D_{1}$ as unknown dynamics will be estimated in this subsection though the feedback control error $e_{r}$, control input $u$, and known function $F$.

Following the previous procedure of designing the estimator in Section 3, we define the filtered variable $e_{r, f}$ with 
respect to $e_{r}$ in order to avoid requiring the information of derivative of control error $\dot{e}_{r}$ in the USDE. We obtain

$$
\begin{gathered}
\kappa \dot{e}_{r, f}+e_{r, f}=e_{r}, \\
e_{r, f}(0)=0 .
\end{gathered}
$$

Then, we can find the following invariant manifold.

Lemma 2. Based on the equation of control error (43), the filtered variables $e_{r, f}, F_{f}$, and $u_{f}$ defined in (22) and (44), the manifold is derived as follows:

$$
\lim _{t \longrightarrow \infty}\left[\lim _{\kappa \longrightarrow 0}\left(\frac{e_{r}-e_{r, f}}{\kappa}+\frac{1}{m_{\mathrm{fc}} C_{p, \mathrm{fc}}}\left(u_{f}+F_{f}\right)-D_{2}\right)\right]=0 .
$$

This manifold is invariant and exponentially attractive for any small positive constant $\kappa>0$.

Proof. we define the auxiliary coordinate for analyzing the manifold invariant:

$$
z_{2}(t)=\frac{e_{r}-e_{r, f}}{\kappa}+\frac{1}{m_{\mathrm{fc}} C_{p, \mathrm{fc}}}\left(u_{f}+F_{f}\right)-D_{2} .
$$

Then, we differentiate (46) with respect to time, that is,

$$
\dot{z}_{2}=\frac{\dot{e}_{r}-\dot{e}_{r, f}}{\kappa}+\frac{1}{m_{\mathrm{fc}} C_{p, \mathrm{fc}}}\left(\dot{u}_{f}+\dot{F}_{f}\right)-\dot{D}_{2}=-\frac{1}{\kappa}\left(z_{2}+\kappa \dot{D}_{2}\right) \text {. }
$$

Moreover, we select $V_{z_{2}}=\left(z_{2}^{2} / 2\right)$ as Lyapunov function to show the bound of $z_{2}$. Thus, the time derivative of $V_{z_{2}}$ is

$$
\begin{aligned}
\dot{V}_{z_{2}} & =z_{2} \dot{z}_{2}=-\frac{1}{\kappa} z_{2}^{2}-\kappa z_{2} \dot{D}_{2} \\
& \leq-\frac{1}{2 \kappa} z_{2}^{2}+\kappa \dot{D}_{1}^{2} \leq-\frac{1}{\kappa} V_{z_{2}}+\kappa \eta_{2}^{2} .
\end{aligned}
$$

Furthermore, (48) is integrated such that we can get $V_{z_{2}}(t) \leq e^{-t / \kappa} V_{z_{2}}(0)+\kappa^{2} \eta_{2}^{2} / 2$. Thus, it is concluded that $z_{2}(t)$ converges exponentially to a small compact around zero, which is expressed as

$$
\left\|z_{2}(t)\right\| \leq \sqrt{z_{2}^{2}(0) e^{-t / \kappa}+\frac{\kappa^{2} \eta_{2}^{2}}{2}}
$$

Following the same analysis in Lemma 1, we can draw the conclusion that $z_{2}$ will converge to zero for $\kappa \longrightarrow 0$ and/ or $\eta_{2} \longrightarrow 0$ along the time. Finally, the manifold $z_{2}=0$ in (45) is invariant and exponentially attractive.

Therefore, the estimator is provided based on the invariant manifold in (45)

$$
\widehat{D}_{2}=\frac{1}{\kappa}\left(e_{r}-e_{r, f}\right)+\frac{1}{m_{\mathrm{fc}} C_{p, \mathrm{fc}}} u_{f}+\frac{1}{m_{\mathrm{fc}} C_{p, \mathrm{fc}}} F_{f} .
$$

Theorem 3. Based on the proposed estimator (50) and the derivative equation of control error (43), the estimation error
$\widetilde{D}_{2}=D_{2}-\widehat{D}_{2}$ will exponentially converge to a small compact set around zero.

Proof. for the analysis purpose, the filtered variable $D_{2, f}$ is defined as

$$
\begin{gathered}
\kappa \dot{D}_{2, f}+D_{2, f}=D_{2}, \\
D_{2, f}(0)=0 .
\end{gathered}
$$

Then, a low-pass filter is applied to the equation (43). We get

$$
\begin{aligned}
\frac{\kappa}{\kappa s+1}\left\{e_{r}\right\}= & -\frac{1}{m_{\mathrm{fc}} C_{p, \mathrm{fc}}}\left(\frac{1}{\kappa s+1}\{u\}+\frac{1}{\kappa s+1}\{F\}\right) \\
& +\frac{1}{\kappa s+1}\left\{D_{2}\right\}+\varepsilon_{2},
\end{aligned}
$$

where the exponentially vanishing term $\varepsilon_{2}$ from the filtered initial value of state $e_{r}(0)$ is neglected.

By substituting (22) and (51), the equation (52) is reformulated as

$$
\dot{e}_{r, f}=\frac{e_{r}-e_{r, f}}{\kappa}=-\frac{1}{m_{\mathrm{fc}} C_{p, \mathrm{fc}}}\left(u_{f}+F_{f}\right)+D_{2, f} .
$$

By the comparison of (46) and (53), we can get the fact $\widehat{D}_{2}=D_{2, f}$. Before we provide the bound of the estimation error $\widetilde{D}_{2}$, the derivative of the estimation error is first computed as follows:

$$
\dot{\widetilde{D}}_{2}=\dot{D}_{2}-\dot{\widehat{D}}_{2}=\dot{D}_{2}-\dot{D}_{2, f}=\dot{D}_{2}-\frac{D-D_{2, f}}{\kappa}=-\frac{1}{\kappa} \widetilde{D}_{2}+\dot{D}_{2} \text {. }
$$

Furthermore, we choose Lyapunov function as $V_{D_{2}}=\left(\widetilde{D}_{2}^{2} / 2\right)$. The derivative of this function is

$$
\begin{aligned}
\dot{V}_{D_{2}} & =\widetilde{D}_{2} \dot{\widetilde{D}}_{2}=-\frac{1}{\kappa} \widetilde{D}_{2}^{2}+\widetilde{D}_{2} \dot{D}_{2} \\
& \leq-\frac{1}{2 \kappa} \widetilde{D}_{2}+\frac{\kappa}{2} \dot{D}_{2}^{2} \leq-\frac{1}{\kappa} V_{D_{2}}+\frac{\kappa}{2} \eta_{2}^{2}
\end{aligned}
$$

Then, the integration of (55) is $V_{D_{2}} \leq\left(e^{-t / \kappa} V_{D_{2}}(0)+\kappa^{2} \eta_{2}^{2} / 2\right)$. Based on this, it can be derived that the estimation error $\widetilde{D}_{2}$ will exponentially converge to a small compact:

$$
\left\|\widetilde{D}_{2}\right\| \leq \sqrt{\widetilde{D}_{2}^{2}(0) e^{-t / \kappa}+\kappa^{2} \eta_{2}^{2}}
$$

Finally, $\widetilde{D}_{2}$ will converge to zero when $\kappa \longrightarrow 0$ and $\eta \longrightarrow \infty$.

4.2. Alternative Composite PI Controller with Unknown Dynamic Compensation. In this section, an alternative composite PI controller is proposed without requiring the derivative of the desired temperature. Since the alternative estimator is designed based on the equation (43), the derivative of the desired temperature $\dot{T}_{f c, r}$ and the unknown dynamics $D_{1}$ are estimated by estimator (56). Thus, the composite PI controller can be designed as 


$$
u=k_{p} e_{r}+k_{i} \int_{0}^{t} e_{r}(\tau) \mathrm{d} \tau+m_{\mathrm{fc}} C_{p, \mathrm{fc}} \widehat{D}_{2}-F .
$$

Then, the following theorem illustrates the convergence of the tracking error and the estimation error for the proposed controller (57).

Theorem 4. To regulate the temperature of the PEMFC system, the control strategy of the composite PI controller (57) is combined with an unknown dynamic compensation (50). The control error $e_{r}$ and the estimation $\widetilde{D}_{2}$ will exponentially converge to a small compact set around zero.

Proof. by substituting (57) into (43), we can get

$$
\dot{e}_{r}=-\frac{1}{m_{\mathrm{fc}} C_{p, \mathrm{fc}}}\left(k_{p} e_{r}+k_{i} e_{\mathrm{ri}}\right)+\widetilde{D}_{2}
$$

where $e_{\mathrm{ri}}=\int_{0}^{t} e_{r}(\tau) \mathrm{d} \tau$ denotes the integral error. To analyze the stability of the controller, the errors including the control error and the integral error are defined as $e=\left[e_{\mathrm{ri}} e_{r}\right]^{T}$. Thus, the equation (58) is reformulated as

$$
\begin{aligned}
\dot{e} & =\left[\begin{array}{c}
\dot{e}_{\mathrm{ri}} \\
\dot{e}_{r}
\end{array}\right]=\left[\begin{array}{cc}
0 & 1 \\
-\frac{k_{i}}{m_{\mathrm{fc}} C_{p, \mathrm{fc}}} & -\frac{k_{p}}{m_{\mathrm{fc}} C_{p, \mathrm{fc}}}
\end{array}\right]\left[\begin{array}{l}
e_{\mathrm{ri}} \\
e_{r}
\end{array}\right]+\left[\begin{array}{l}
0 \\
1
\end{array}\right] \widetilde{D}_{2} \\
& =A_{2} e+B_{2} \widetilde{D}_{2} .
\end{aligned}
$$

Moreover, there are symmetric and positive definite matrices $P_{2}$ and $Q_{2}$ such that the matrix $A_{2}$ fulfills the equation $P_{2} A_{2}+A_{2}^{T} P_{2}=-Q_{2}$. Then, we choose the Lyapunov function as $V_{e 2}=\left(\left(e^{T} P_{2} e / 2\right)+\left(\widetilde{D}_{2}^{2} / 2\right)\right)$. Its derivative is expressed as

$$
\begin{aligned}
\dot{V}_{e 2} & =\frac{1}{2} e^{T} P_{2} \dot{e}+\frac{1}{2} e^{T} P_{2} e+\widetilde{D}_{2} \dot{\widetilde{D}}_{2} \\
& =\frac{1}{2} e^{T} P_{2} A_{2} e+\frac{1}{2} e^{T} A_{2}^{T} P_{2} e+e^{T} P_{2} B_{2} \widetilde{D}_{2}+\widetilde{D}_{2} \dot{\widetilde{D}}_{2} \\
& =-\frac{1}{2} e^{T} Q_{2} e+e^{T} P_{2} B_{2} \widetilde{D}_{2}-\frac{1}{\kappa} \widetilde{D}_{2}^{2}+\widetilde{D}_{2} \dot{D}_{2} .
\end{aligned}
$$

Based on Young's inequality with a positive constant $m>0$, we can further derive that

$$
\begin{aligned}
\dot{V}_{e 2} & \leq-\frac{1}{2}\left(\lambda_{\min }\left\{Q_{2}\right\}-\frac{m}{2}\right)\|e\|^{2}-\left(\frac{1}{\kappa}-\frac{1+\left\|P_{2} B_{2}\right\|^{2}}{2 m}\right) \widetilde{D}_{2}^{2}+\frac{m \eta_{2}^{2}}{2} \\
& \leq-\psi_{2} V+\zeta_{2},
\end{aligned}
$$

where there are positive constants of $\psi_{2}=\min \left\{\left(\lambda_{\min }\left\{Q_{2}\right\}-\right.\right.$ $\left.(m / 2)) / \lambda_{\max }\left\{P_{2}\right\}, 2\left(1 / \kappa-\left(1+\left\|P_{2} B_{2}\right\|^{2}\right)\right) /(2 m)\right\} \quad$ and $\zeta_{1}=\left(m \eta_{2}^{2} / 2\right)$ when constants $m$ and $\kappa$ are satisfied, $m \leq 2 \lambda_{\min }\left\{Q_{2}\right\}$ and $\kappa \leq 2 m /\left(1+\left\|P_{2} B_{2}\right\|^{2}\right)$; the maximum eigenvalue of the matrix $P_{2}$ is denoted as $\lambda_{\max }\left\{P_{2}\right\}$ and $\lambda_{\min }\left\{Q_{2}\right\}$ is the minimum eigenvalue of the matrix $Q_{2}$. Then, we can further derive $V(t) \leq e^{-\psi_{2} t} V(0)+\zeta_{2} / \psi_{2}$, which implies that the control error $e$ and the estimation error $\widetilde{D}_{2}$ will exponentially converge to the following small compact set around zero:

$$
\Omega:=\left\{e, \widetilde{D}_{2} \mid\|e\| \leq \sqrt{\frac{\zeta_{2}}{\psi_{2}}},\left\|\widetilde{D}_{2}\right\| \leq \sqrt{\frac{\zeta_{2}}{\psi_{2}}}\right\} .
$$

\section{Simulation Results}

In this section, the proposed control strategy for the PEMFC system is conducted in the MATLAB/Simulink environment. For the simulation of PEMFC, the stoichiometric ratios of oxygen and hydrogen are set as $K_{\mathrm{O}_{2}}=4$ and $K_{\mathrm{H}_{2}}=2.5$, respectively. And the current of fuel cell is set as $I_{\mathrm{fc}}=15 \mathrm{~A}$, where the cooling fan cannot remove enough heat to maintain the temperature of PEMFC. Moreover, the disturbance is set as a bounded signal $d(t)=0.005 \sin (0.1 t) e^{(-0.001 t)}+0.005$. The temperature of the humidifier at the anode side is set as $T_{\text {hum,an }}^{\text {set }}=40^{\circ} \mathrm{C}$. The initial temperature of the cathode humidifier is $34.5^{\circ} \mathrm{C}$. The ideal operation temperature of the humidifier at the cathode side is set as $T_{\text {hum,ca }}^{\text {set }}=29.5^{\circ} \mathrm{C}$. The temperature profile of the cathode humidifier in the simulation is depicted in Figure 4. It is illustrated that the humidifier performs a slow dynamics of reducing its temperature to reach the idea operation temperature. Due to lack of the effective cooling system, the humidifier takes almost $2000 \mathrm{~s}$ to reduce its temperature, which implies that reducing the temperature of input gases is not effective enough to regulate PEMFC temperature. The temperature of line heaters is chosen as the average value between the humidifier temperature with the desired PEMFC temperature, which fulfills the purpose of line heaters to maintain the gas temperature in the pipelines.

Based on the USDE in (29) and (50), only one tuning constant is set as $\kappa=10^{-3}$. For the PI controller, the gain coefficients are $k_{p}=4$ and $k_{i}=1$, which are determined based on the tuning rule in the frequency domain [27] in order to maintain the stable performance. The comparison of temperature profiles regulated by the proposed composite PI controllers ((37) and (57)) and the classical PI is shown in Figure 5. It is illustrated that the controllers ((37) and (57)) and the classical PI can achieve the temperature regulation. In the steady-state, the PEMFC temperature can reach to the desired value by the controllers. However, it is noted that the composite PI control performs much better than the classical PI control in the transient performance. The control errors in the composite PI controllers are small and bounded. Moreover, the convergence speed of the composite PI controllers is faster than that of the classical PI controller.

Before the control input enters into the system, the antiwindup scheme is used to avoid the input mass flows in the negative value or over the system bounds. The control input $u$ of the total energy is given in Figure 6. In practice, the additional mass flow of the air is considered as a practical control input. Based on the relation in (19), the additional mass flow of the air can be obtained and the profile of the additional air mass flow is depicted in Figure 7. It is shown 


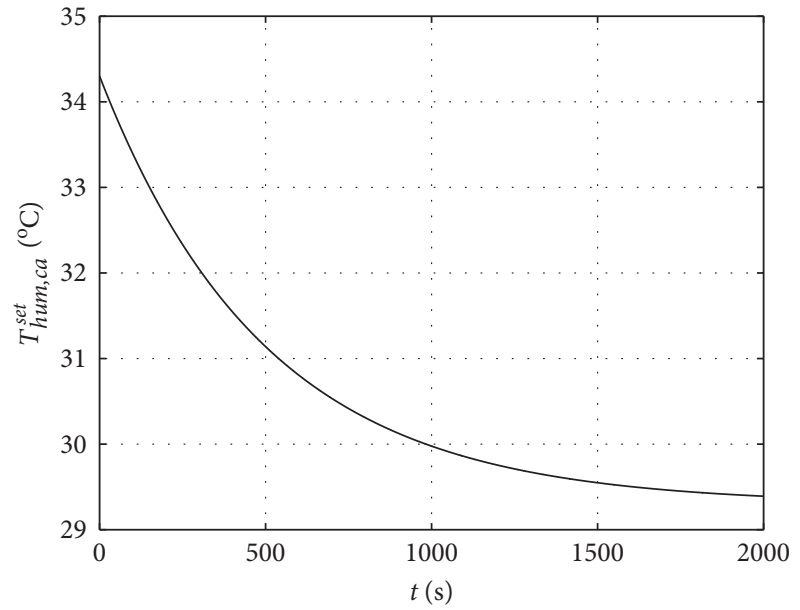

Figure 4: The profile of the humidifier temperature at the cathode side.

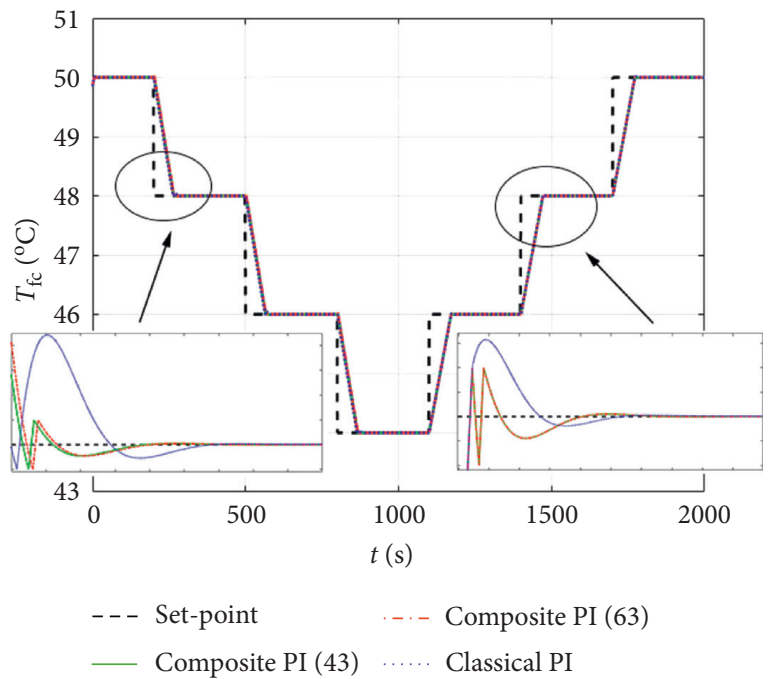

Figure 5: The temperature profiles regulated by the proposed composite PI controller ((37) and (57)) and the classical PI.

that the anti-windup has a good performance such that the additional mass flow of the air cannot exceed the system constraint.

Since the mass flow of the air is changed during the control process, the partial pressure of oxygen will be changed which has an impact on the PEMFC voltage of (10). Moreover, there are many variables in the PEMFC voltage related to the temperature, which has been discussed in (12)-(15). The voltage profile affected by the proposed composite PI controllers ((37) and (57)) and the classical PI is depicted in Figure 8, which implies that the proposed control strategy has a small impact to the PEMFC voltage. Besides, the profile of the cooling energy generated by fan 1 is shown in Figure 9. It is noticed that the fan turns on when the PEMFC temperature reduces. Thus, there are no coupling effects between the controller and the fan operation mode. Figure 10 shows the result profile of estimated unknown dynamics $D_{1}$ and $D_{2}$. It is illustrated that the USDE

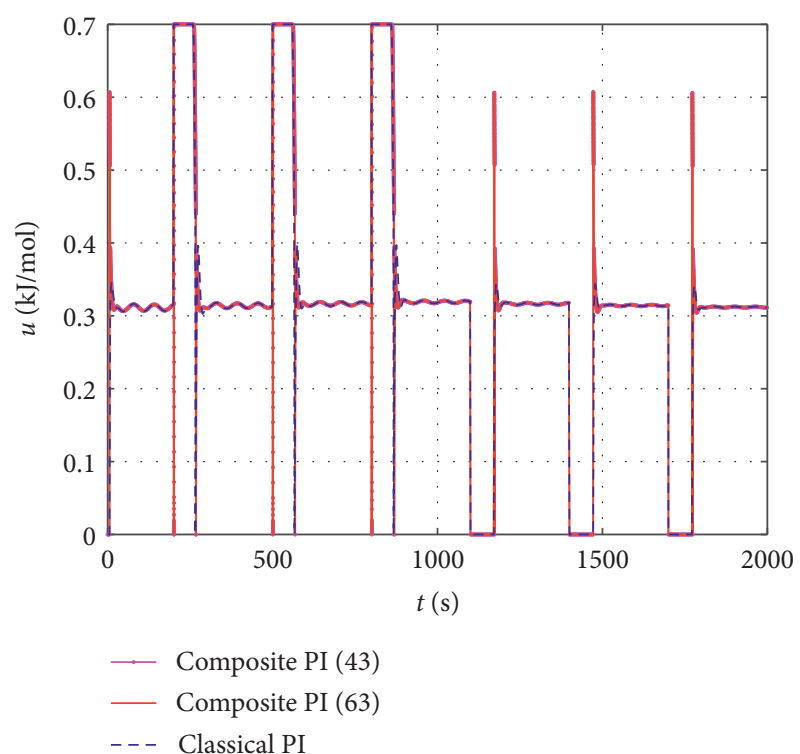

FIgUre 6: The profiles of the control input by classical PI and composite PI ((37) and (57)).

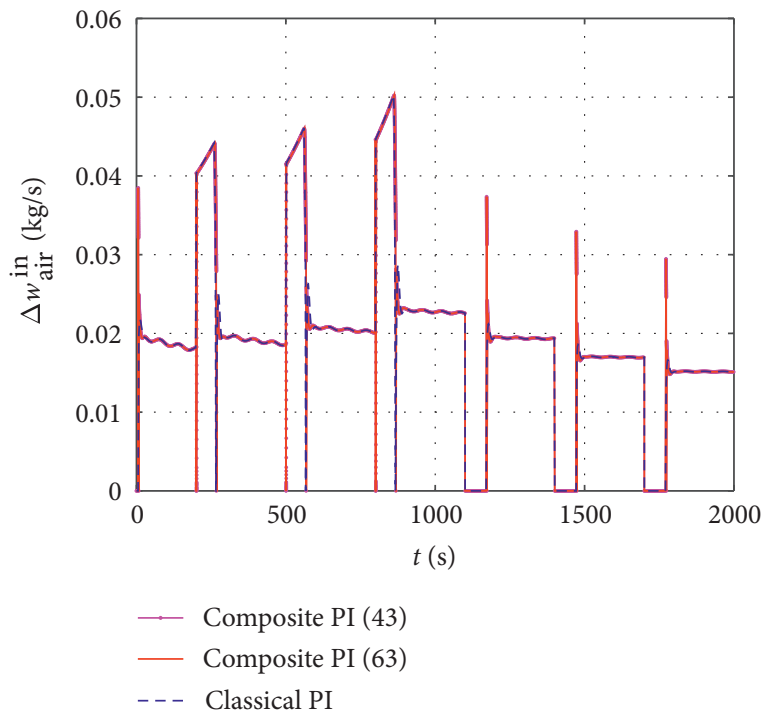

FIgURE 7: The profiles of the additional air mass flows by classical PI and composite PI ((37) and (57)).

can achieve accurate estimation. There are some sharp transient peaks in $D_{2}$, which are affected by the change of the desired temperature and the fan operation condition.

For the presented simulation results above, the antiwindup scheme plays an important role in the proposed control strategy. In order to further show the efficacy of composite PI controllers, the amplitude of the desired temperature is set as a small variation. The control input $u$ for this case is given in Figure 11. It is shown that the unknown system dynamics estimators ((29) and (50)) can compensate the unknown dynamics in the composite PI controllers ((37) and (57)). The regulated temperature profiles by the proposed composite PI controllers ((37) and (57)) and the classic PI controller are depicted in Figure 12. 


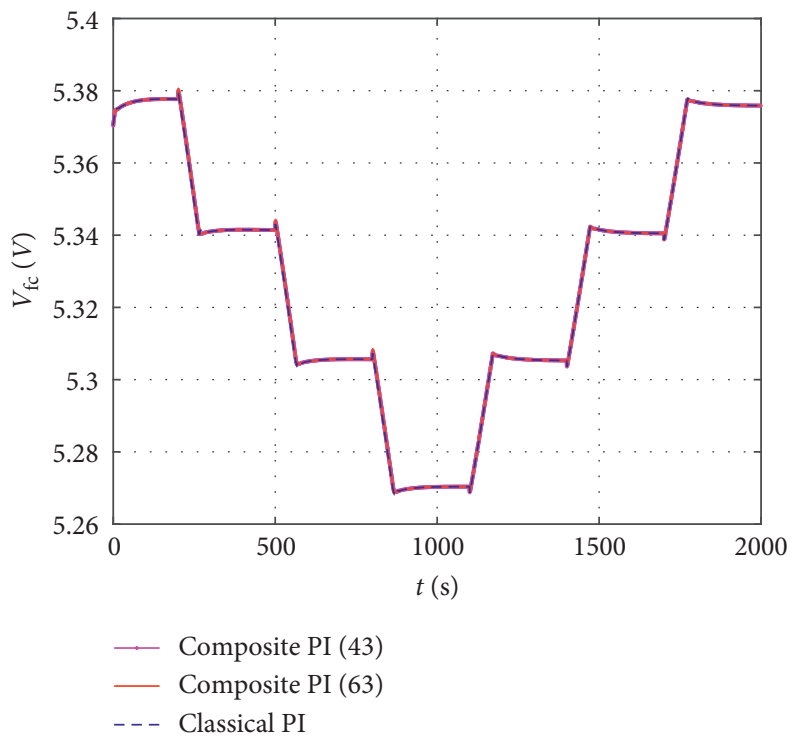

Figure 8: The voltage profiles regulated by the proposed composite PI controllers ((37) and (57)) and the classical PI.

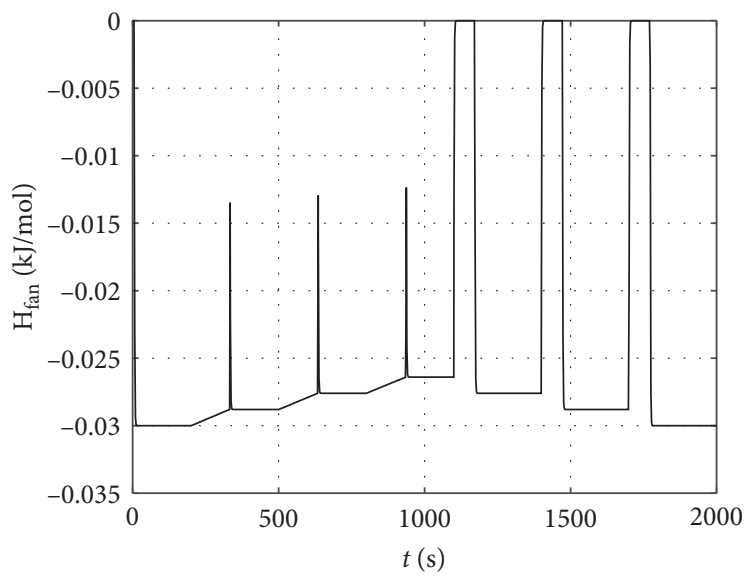

FIGURE 9: Simulation profile of the cooling energy by fan 1.

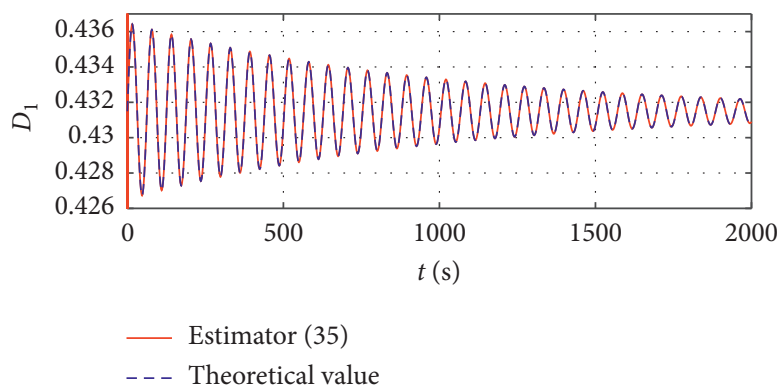

(a)

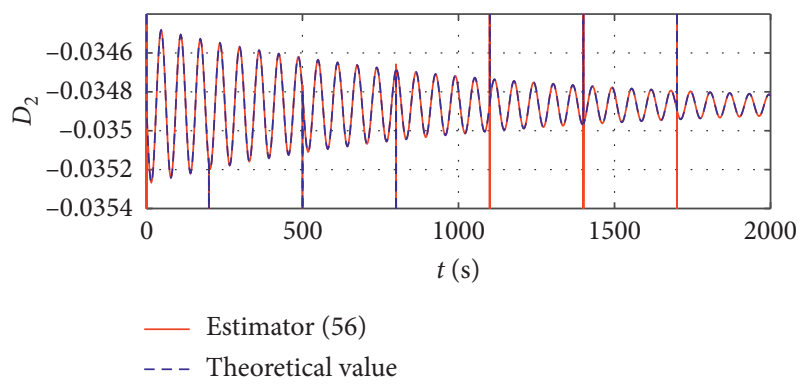

(b)

Figure 10: Estimation result of the unknown dynamics $D_{1}$ and $D_{2}$ by the estimators $((29)$ and (50)). 


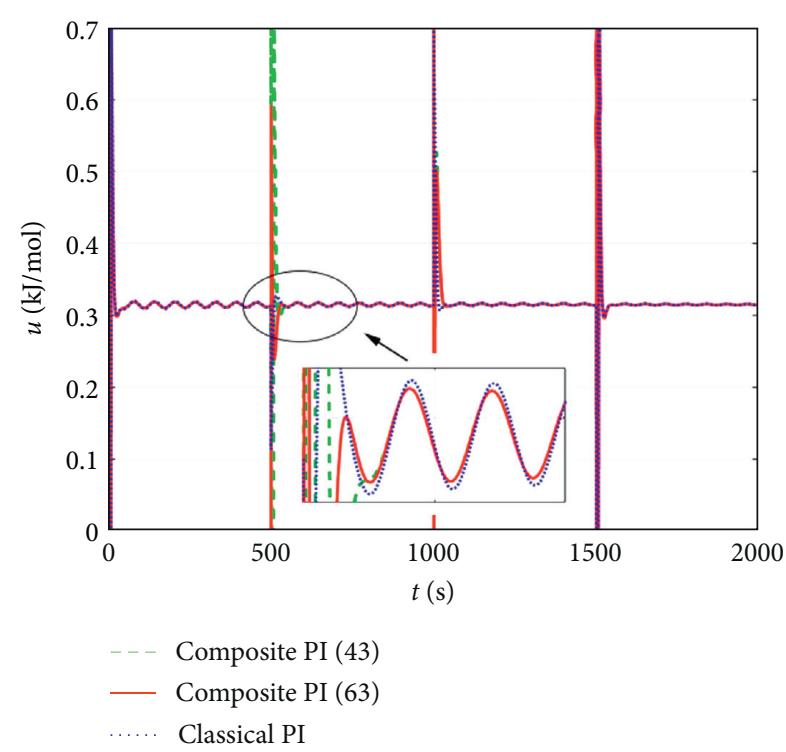

FIgURE 11: The profiles of the control input by classical PI and composite PI ((37) and (57)) for the small variation of temperature regulation.

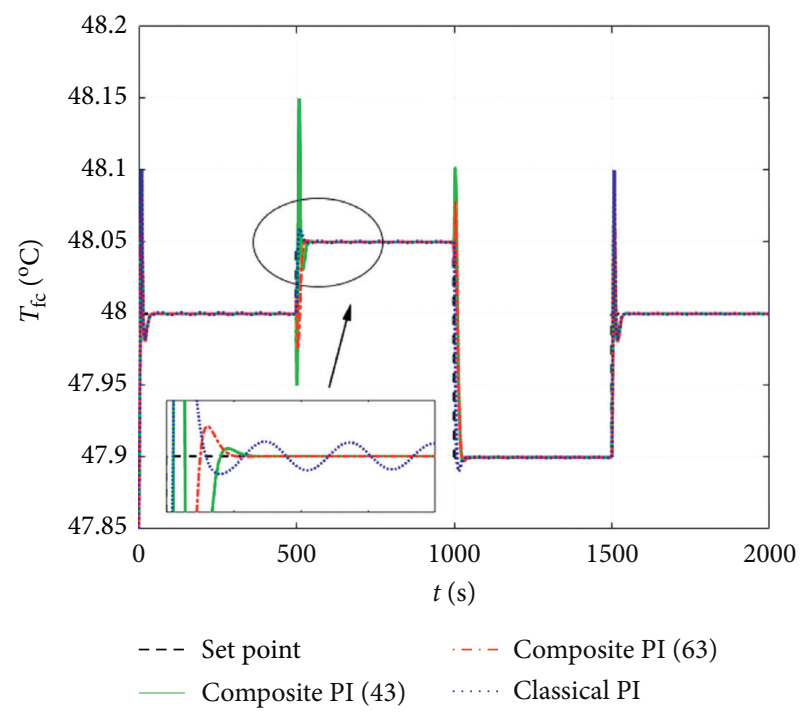

FIGURE 12: The small variation of temperature regulation profiles by the proposed composite PI controller ((37) and (57)) and the classical PI.

It is shown that the temperature errors by the composite PI controllers ((37) and (57)) are always small while the result by the classic PI controller is affected by the unknown dynamics $D_{1}$ and $D_{2}$ such that the temperature cannot be regulated to the desired temperature.

\section{Conclusions}

In this paper, a composite PI control with the USDE is proposed for a PEMFC system. Considering the limitation of the cooling fan system, an alternative control strategy is developed by reducing the temperature of input air through the humidifier and simultaneously increasing the mass flow of air in order to eliminate the excess heat that a cooling fan cannot remove. Then, a low-pass filter is applied to the system in order to obtain an invariant manifold. This invariant manifold contains the information between the unknown dynamics with the known variables, which can be directly used to construct the estimator. Moreover, the requirement of the state derivative can be avoided which is suitable for practical application. The derived estimator can ensure the exponential convergence and is easily incorporated into the proposed control strategy such that the performance of the PI control is improved and the effect of unknown dynamics can be eliminated. Finally, numerical simulations of the PEMFC system are provided. The simulation results demonstrate the efficacy of the proposed control strategy and the improvement of control performance.

\section{Data Availability}

The data used in this paper are available from the corresponding author upon request.

\section{Conflicts of Interest}

The authors declare that there are no conflicts of interest regarding the publication of this paper.

\section{Acknowledgments}

This work was partially funded by the Spanish National Project DOVELAR (ref. RTI2018-096001-B-C32), the AGAUR of Generalitat de Catalunya through the Advanced Control Systems (SAC) group grant (2017 SGR 482), and the Spanish State Research Agency through the María de Maeztu Seal of Excellence to IRI (MDM-2016-0656). This work was partially funded by the Chinese Scholarship Council (CSC) under grant (201808390007). This work was partially funded by National Natural Science Foundation of China under Grants 61922037 and 61873115.

\section{References}

[1] F. Barbir, PEM Fuel Cells: Theory and Practice, Academic Press, Cambridge, MA, USA, 2012.

[2] J.-L. Casteleiro-Roca, A. J. Barragán, F. Segura, J. L. CalvoRolle, and J. M. Andújar, "Fuel cell output current prediction with a hybrid intelligent system," Complexity, vol. 2019, Article ID 6317270, 10 pages, 2019.

[3] M. H. Khooban, N. Vafamand, and J. Boudjadar, "Tracking control for hydrogen fuel cell systems in zero-emission ferry ships," Complexity, vol. 2019, Article ID 5358316, 9 pages, 2019.

[4] J. Wu, X. Z. Yuan, J. J. Martin et al., "A review of pem fuel cell durability: degradation mechanisms and mitigation strategies," Journal of Power Sources, vol. 184, no. 1, pp. 104-119, 2008.

[5] A. Cecilia and R. Costa-Castelló, "Observador de alta ganancia con zona muerta ajustable para estimar la saturación de agua líquida en pilas de combustible tipo PEM," Revista Iberoamericana de Automática e Informática industrial, vol. 17, no. 2, pp. 169-180, 2020. 
[6] S. G. Kandlikar and Z. Lu, "Thermal management issues in a PEMFC stack - a brief review of current status," Applied Thermal Engineering, vol. 29, no. 7, pp. 1276-1280, 2009.

[7] R. Banerjee and S. G. Kandlikar, "Two-phase flow and thermal transients in proton exchange membrane fuel cells - a critical review," International Journal of Hydrogen Energy, vol. 40, no. 10, pp. 3990-4010, 2015.

[8] S. Strahl, A. Husar, P. Puleston, and J. Riera, "Performance improvement by temperature control of an open-cathode pem fuel cell system," Fuel Cells, vol. 14, no. 3, pp. 466-478, 2014.

[9] Y. Saygili, I. Eroglu, and S. Kincal, "Model based temperature controller development for water cooled pem fuel cell systems," International Journal of Hydrogen Energy, vol. 40, no. 1, pp. 615-622, 2015.

[10] Y.-X. Wang, F.-F. Qin, K. Ou, and Y.-B. Kim, “Temperature control for a polymer electrolyte membrane fuel cell by using fuzzy rule," IEEE Transactions on Energy Conversion, vol. 31, no. 2, pp. 667-675, 2016.

[11] F. Chen, Y. Yu, and Y. Gao, "Temperature control for proton exchange membrane fuel cell based on current constraint with consideration of limited cooling capacity," Fuel Cells, vol. 17, no. 5, pp. 662-670, 2017.

[12] S. Komada, M. Ishida, K. Ohnishi, and T. Hori, "Disturbance observer-based motion control of direct drive motors," IEEE Transactions on Energy Conversion, vol. 6, no. 3, pp. 553-559, 1991.

[13] Y. Oh and W. K. Chung, "Disturbance-observer-based motion control of redundant manipulators using inertially decoupled dynamics," IEEE/ASME Transactions on Mechatronics, vol. 4, no. 2, pp. 133-146, 1999.

[14] W.-H. Chen, "Disturbance observer based control for nonlinear systems," IEEE/ASME Transactions on Mechatronics, vol. 9, no. 4, pp. 706-710, 2004.

[15] J. Q. Han, "The extended state observer of a class of uncertain systems," Control and Decision, vol. 10, no. 1, pp. 85-88, 1995.

[16] B.-Z. Guo and Z.-1. Zhao, "On the convergence of an extended state observer for nonlinear systems with uncertainty," Systems \& Control Letters, vol. 60, no. 6, pp. 420-430, 2011.

[17] D. Li, C. Li, Z. Gao, and Q. Jin, "On active disturbance rejection in temperature regulation of the proton exchange membrane fuel cells," Journal of Power Sources, vol. 283, pp. 452-463, 2015.

[18] W. Xue, X. Zhang, L. Sun, and H. Fang, "Extended state filter based disturbance and uncertainty mitigation for nonlinear uncertain systems with application to fuel cell temperature control," IEEE Transactions on Industrial Electronics, vol. 67, no. 12, pp. 10682-10692, 2020.

[19] J. Na, J. Yang, S. Wang, G. Gao, and C. Yang, "Unknown dynamics estimator-based output-feedback control for nonlinear pure-feedback systems," in IEEE Transactions on Systems, Man, and Cybernetics: SystemsIEEE, Piscataway, NJ, USA, 2019.

[20] J. Na, Y. Li, Y. Huang, G. Gao, and Q. Chen, "Output feedback control of uncertain hydraulic servo systems," IEEE Transactions on Industrial Electronics, vol. 67, no. 1, pp. 490-500, 2019.

[21] J. Na, B. Jing, Y. Huang, G. Gao, and C. Zhang, "Unknown system dynamics estimator for motion control of nonlinear robotic systems," IEEE Transactions on Industrial Electronics, vol. 67, no. 5, pp. 3850-3859, 2019.

[22] J. Na, A. S. Chen, Y. Huang et al., "Air-fuel ratio control of spark ignition engines with unknown system dynamics estimator: theory and experiments," in IEEE Transactions on Control Systems TechnologyIEEE, Piscataway, NJ, USA, 2019.
[23] J. T. Pukrushpan, A. G. Stefanopoulou, and H. Peng, Control of Fuel Cell Power Systems: Principles, Modeling, Analysis and Feedback Design, Springer Science \& Business Media, Berlin, Germany, 2004.

[24] R. M. Felder, R. W. Rousseau, and L. G. Bullard, Elementary Principles of Chemical Processes, Wiley, Hoboken, NJ, USA, 4th edition, 2015.

[25] C. Kunusch, P. F. Puleston, M. A. Mayosky, and A. P. Husar, "Control-oriented modeling and experimental validation of a pemfc generation system," IEEE Transactions on Energy Conversion, vol. 26, no. 3, pp. 851-861, 2011.

[26] J. Liu, Y. Gao, X. Su, M. Wack, and L. Wu, "Disturbanceobserver-based control for air management of pem fuel cell systems via sliding mode technique," IEEE Transactions on Control Systems Technology, vol. 27, no. 3, pp. 1129-1138, 2018.

[27] K. J. Åström and R. M. Murray, Feedback Systems: An Introduction for Scientists and Engineers, Princeton university press, Princeton, NJ, USA, 2010. 